\title{
Development of a Layer-by-Layer Assembled Film on Hydrogel for Ocular Drug Delivery
}

\author{
Pin Chen, Xin Wang, Yan Dong, and Xiaohong Hu \\ School of Material Engineering, Jinling Institute of Technology, Nanjing 211169, China \\ Correspondence should be addressed to Xiaohong Hu; hxh@jit.edu.cn
}

Received 5 November 2014; Accepted 8 December 2014

Academic Editor: Yen-Chih Lin

Copyright ( 2015 Pin Chen et al. This is an open access article distributed under the Creative Commons Attribution License, which permits unrestricted use, distribution, and reproduction in any medium, provided the original work is properly cited.

\begin{abstract}
Hydrogel is a kind of attractive drug carriers because of its good biocompatibility and transparency. But traditional hydrogel showed some restrictions in its application in ocular drug delivery. A simple surface modification technique based on layer-by-layer (LbL) self-assembled multilayer for ocular drug delivery was developed in this work. Polycarboxymethyl- $\beta$-cyclodextrin (poly(CM- $\beta$ $\mathrm{CD})$ )/poly-l-lysine (PLL) multilayer film was designed and constructed for ocular drug delivery, since $\beta$-CD showed good drug delivery property. The properties such as the contact angle and transparency varied a little with the deposition of poly(CM- $\beta$ CD)/PLL multilayer. Orfloxacin and puerarin were loaded into multilayer during the self-assembly procedure by two methods, which were tracked by the largest drug absorbance of UV spectrum. The loaded drug amount by incorporating drugs into poly(CM$\beta$-CD) solution was larger than that by incorporating drugs into PLL solution. The loaded drug in the multilayer could gradually be released from multilayer in some period either for orfloxacin or for puerarin. The drug release behavior was influenced by drug loading method and $\mathrm{pH}$ value of released medium. Moreover, the balanced released drug amount by incorporating drugs into poly $(\mathrm{CM}-\beta-\mathrm{CD})$ solution is much smaller than that by incorporating drugs into PLL solution.
\end{abstract}

\section{Introduction}

Poor ocular bioavailability and short action duration of eye drugs commonly trouble both patients and doctors. With the development of drug delivery system, the bioavailability is improved and the action duration is prolonged when ophthalmic drug is delivered by drug delivery system [14]. Therefore, the curative effects of ophthalmic drug would be enhanced. Hydrogel is a kind of attractive drug carriers because of its biocompatibility [5-7]. Moreover, hydrogel had good transparency, which made it have wide applications in ophthalmology [8-12]. However, traditional copolymer hydrogel as drug carrier had disadvantages of limited drug loading amount and burst release in our previous research work [13]. In order to solve this problem, we attempted to enhance the interaction between drug and hydrogel network by introduction of functional chitosan derivatives and water soluble graphene oxide $[14,15]$. Though the method succeeded to a certain extent, it brought some new problems such as limited light transmittance. Therefore, some measures of hydrogel bulk design or hydrogel surface modification for ocular drug delivery are also needed.

Layer-by-layer (LbL) self-assembly technique is a simple and commonly used method to realize surface modification, which has attracted much attention because of its simplicity in procedure, wide choice of materials, and fine-tuning of the microstructure [16-21]. LbL assembled multilayer is fabricated by sequential adsorption of materials with complementary functional groups employing electrostatic interactions, hydrogen bonding, or covalent interactions [1621]. The electrostatic interaction between cationic and anionic polyelectrolyte multilayer (PEM) is intensively used [1921]. In our previous work, chitosan (CS)/hyaluronic acid (HA) multilayer is constructed on contact lenses using LbL technique to increase the hydrophilicity and reduce protein deposition as well as to deliver ophthalmic drug, but burst drug release within 2 hours still occurred [22]. Therefore, in 


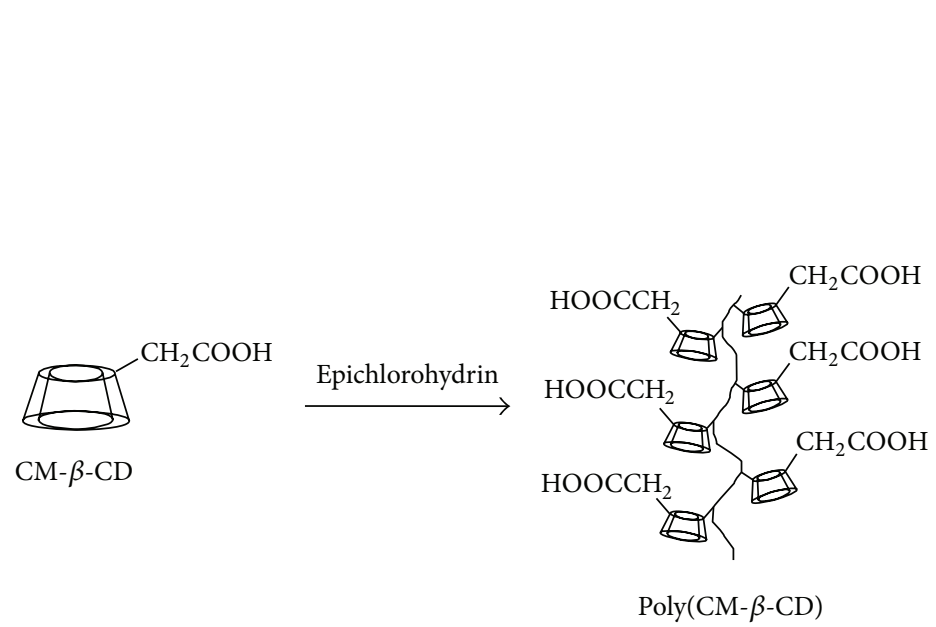

(a)

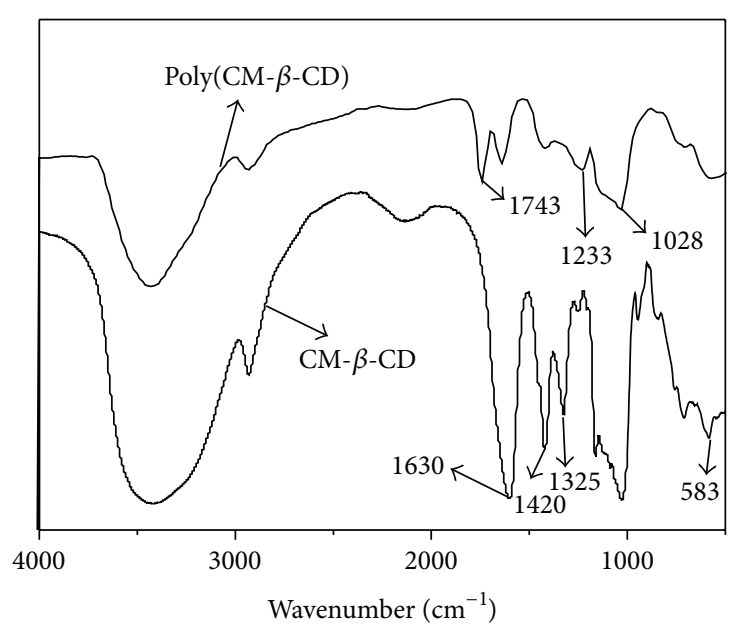

(b)

FIGURE 1: (a) Schematic illustration to show the synthesis of poly $(C M-\beta-C D)$. (b) IR spectra of CM- $\beta$-CD and poly(CM- $\beta-C D)$.

order to realize drug delivery, new PEMs are required to be designed for ocular drug delivery.

As far as we know, a group of cyclic oligosaccharides with a hydrophobic cavity can form inclusion complex with small molecules [23-25]. $\beta$-Cyclodextrin $(\beta-C D)$ is one of cyclic oligosaccharides, which has exhibited good properties to control drug release [26-28]. Thus, $\beta$-CD has designed many kinds of carriers [26-28]. In order to satisfy the need of different kinds of drug carriers, many $\beta$-CD derivatives have been synthesized including carboxymethyl- $\beta$-cyclodextrin (CM$\beta$-CD). Since $\beta$-CD has been proven to be a good material of drug carriers, a polyanion based on $\beta$-CD was designed and synthesized using CM- $\beta$-CD in the work. Moreover, polyl-lysine (PLL) was chosen as a polycation due to its good biocompatibility and wide application in the field of drug delivery and tissue engineering. Furthermore, commonly used eye drugs, orfloxacin, and puerarin were loaded into PEMs during assembly procedure using different method. Finally, the drug release behaviors in different medium were investigated in detail.

Though LBL multilayers have been intensively studied, these researches focused on fundamental properties [29-32]. The investigations of their practical applications in many fields are also needed. The $\mathrm{p}(\mathrm{CM}-\beta-\mathrm{CD}) / \mathrm{PLL}$ in this work was first designed and constructed, aiming at ocular drug delivery. The results of the research would help us to widen the application of multilayer.

\section{Experimental}

2.1. Materials. Hyaluronic acid (HA) and hydroxyethyl methacrylate (HEMA) were obtained from Shanghai Jingchun Industries Co. Ltd., China, and distilled under vacuum before use. Carboxymethyl- $\beta$-cyclodextrin (CM- $\beta$-CD) was obtained from Shandong Binzhou Zhiyuan BioTechnology Co., Ltd.; epichlorohydrin, ammonium persulphate (APS), and $\mathrm{N}, \mathrm{N}, \mathrm{N}^{\prime}, \mathrm{N}^{\prime}$-tetramethylethylenediamine (TEMED) were obtained from Shanghai Chemical Industries
Co. Ltd. (China). Poly-l-lysine (PLL) was obtained from Sigma. Orfloxacin and puerarin were purchased from Zhengzhou Andrew Biological Engineering Co., Ltd., China. All other reagents and solvents were of analytical grade and were used as received.

2.2. Preparation of Hydrogel. $2.7 \mathrm{~mL}$ of water was added into $5 \mathrm{~mL}$ HEMA, to which certain amounts of APS and TEMED with equal molar ratio were, respectively, added with final initiator concentration at $0.5 \% .500 \mu \mathrm{L}$ of the above mixture was injected into a circle model ( $200 \mu \mathrm{m}$ thickness), which was then input into oven at $60^{\circ} \mathrm{C}$. $1 \mathrm{~h}$ later, the formed hydrogel was obtained. The dried hydrogel was put in $20 \mathrm{~mL}$ epichlorohydrin solution, to which $2.8 \mathrm{~mL} 0.55 \mathrm{M}$ sodium hydroxide was added. The hydrogel was transferred to $13.5 \mathrm{~g} / \mathrm{L}$ HA solution, after the reaction had lasted for $5 \mathrm{~h}$. HA was grafted onto hydrogel surface after the reaction had lasted another $10 \mathrm{~h}$ at $85^{\circ} \mathrm{C}$. HA modified hydrogel was formed as a multilayer substrate.

2.3. Synthesis of Poly $(C M-\beta-C D) .2 \mathrm{~g}$ CM- $\beta-C D$ was dissolved in $10 \mathrm{~mL} 30 \% \mathrm{NaOH}$ solution. The solution was heated to $40^{\circ} \mathrm{C}$, to which $4 \mathrm{~mL}$ epichlorohydrin was slowly added. Further, the mixture was heated to $60^{\circ} \mathrm{C}$. When the solution became viscous, another $36 \%-38 \% \mathrm{HCl}$ was added to adjust the $\mathrm{pH}$ value of 7 . The resultant mixture was sealed in a membrane with a cut-off molecular weight of $10 \mathrm{kDa}$ and dialyzed in a large amount of triple-distilled water for 3 months. The white solid was obtained by freeze-drying and ground with $\mathrm{KBr}$. Then they were made tablet and characterized by infrared spectroscopy (IR, Nicolet IS10).

2.4. Poly(CM- $\beta-C D) / P L L$ Multilayer Deposition and Characterization. Two polyelectrolytes were dissolved in $\mathrm{NaCl}$ solution $(0.15 \mathrm{M})$ with the final concentration of $1 \%(\mathrm{w} / \mathrm{v})$. Self-assembled multilayer on hydrogel was performed manually. After swelling, hydrogel was gently rinsed with $0.15 \mathrm{M}$ 


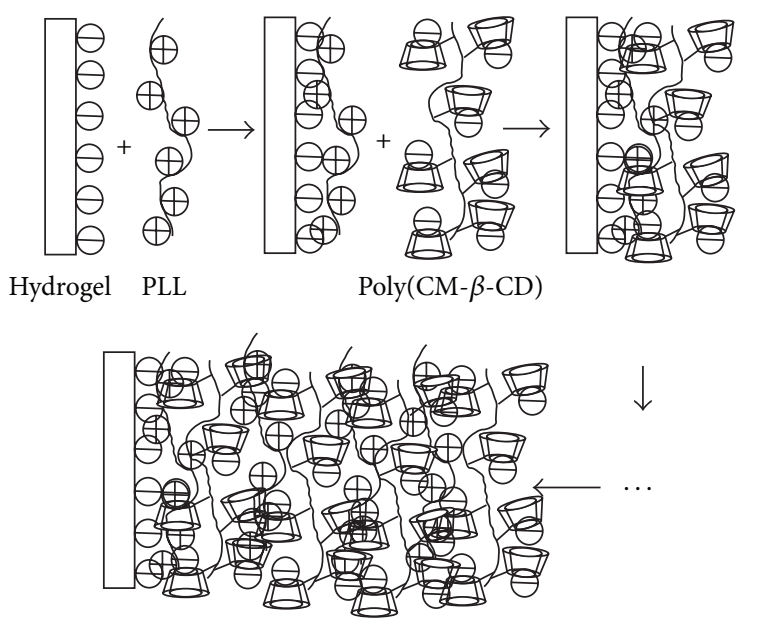

(a)

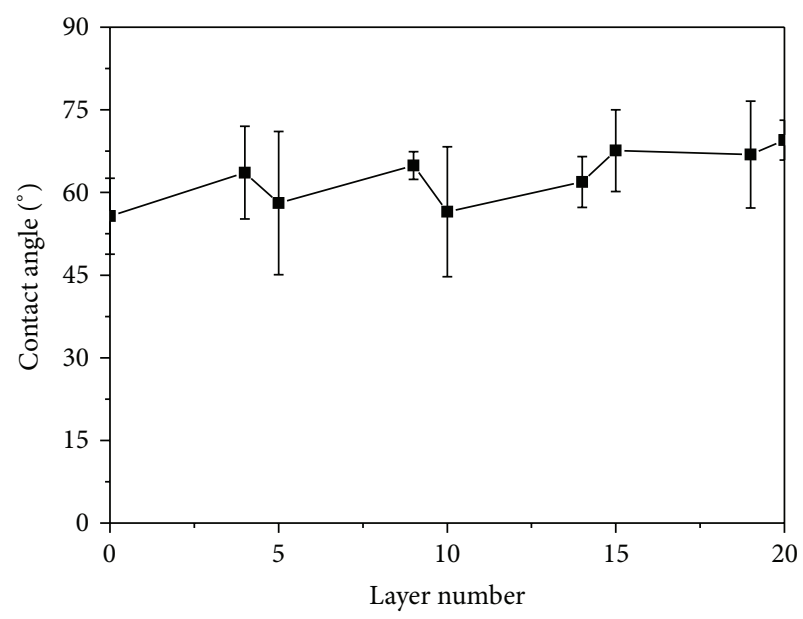

(b)

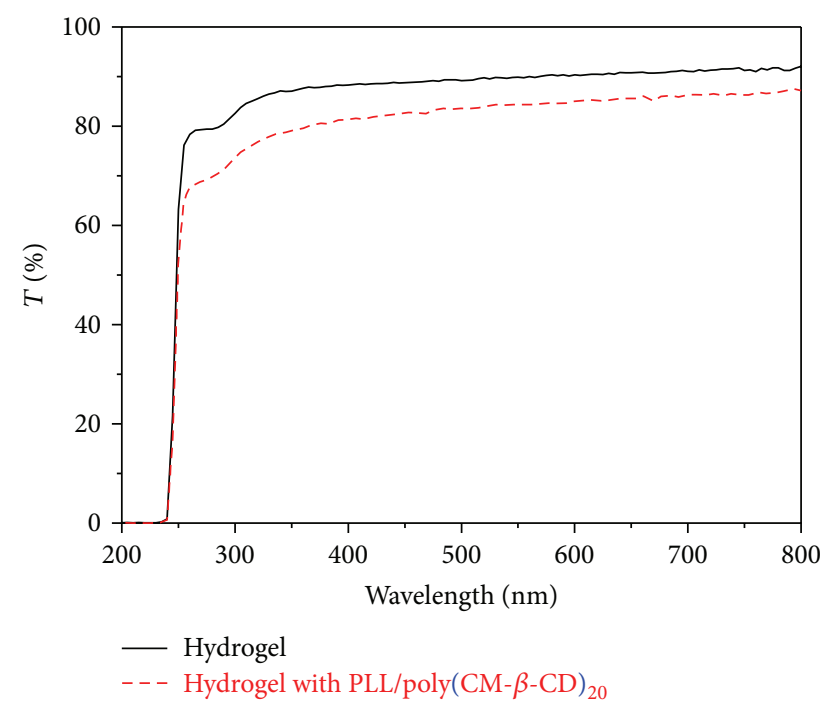

(c)

FIGURE 2: (a) Schematic illustration to show self-assembly process of poly(CM- $\beta$-CD)/PLL multilayer on hydrogel. (b) Contact angle of hydrogel with assembly of poly(CM- $\beta-\mathrm{CD}) / \mathrm{PLL}$ multilayer. (c) Light transmittance of hydrogel and hydrogel with poly $(\mathrm{CM}-\beta-\mathrm{CD}) / \mathrm{PLL}{ }_{20}$ multilayer.

$\mathrm{NaCl}$ and immerged in PLL solution for $20 \mathrm{~min}$. Hydrogel was then rinsed in $0.15 \mathrm{M} \mathrm{NaCl}$ solution and subsequently immerged in poly $(\mathrm{CM}-\beta-\mathrm{CD})$ solution for $20 \mathrm{~min}$, followed by rinsing in $0.15 \mathrm{M} \mathrm{NaCl}$ solution again. After each step, the hydrogel was lightly blown to remove residual liquid. This procedure was repeated to assemble poly $(\mathrm{CM}-\beta-\mathrm{CD}) / \mathrm{PLL}$ multilayer on hydrogel. Poly(CM- $\beta$-CD)/PLL multilayer was characterized by UV spectroscopy (Cary 50) and contact angle measurement system (OCA35).

2.5. The Drug Assembling in Poly $(C M-\beta-C D) / P L L$ Multilayer. Drug was loaded in poly $(\mathrm{CM}-\beta-\mathrm{CD}) / \mathrm{PLL}$ multilayer assembly procedure using orfloxacin and puerarin as model drugs. Drugs were introduced into poly $(\mathrm{CM}-\beta-\mathrm{CD})$ solution or PLL solution with final drug concentration at $1 \mathrm{mg} / \mathrm{mL}$, respectively. 20 layers of poly(CM- $\beta$-CD)/PLL were built up on hydrogel using LBL technique as mentioned above. The assembly procedure was tracked by UV spectroscopy (Cary $50)$.

2.6. Drug Releasing Behavior. The hydrogels with drug were submerged in $4 \mathrm{~mL}$ medium. Every certain time, $2 \mathrm{~mL}$ released solution was moved and $2 \mathrm{~mL}$ fresh medium was added at the same time. The released solution was diluted to $4 \mathrm{~mL}$. The absorbance of diluted drug solution at $285 \mathrm{~nm}$ for orfloxacin or $255 \mathrm{~nm}$ for puerarin was recorded by UV spectroscopy. The released drug concentration was obtained by referring to a calibration curve, which was constructed from known concentrations of drug solutions. Then the 

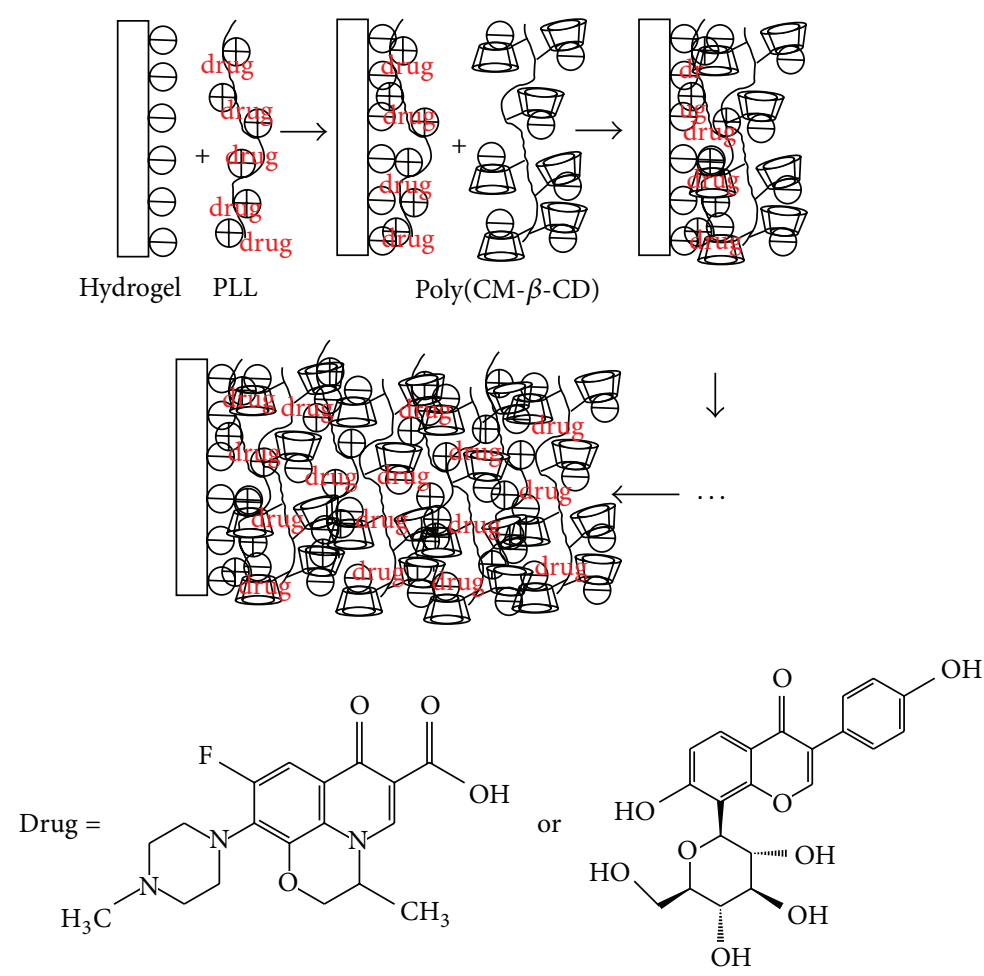

(a)

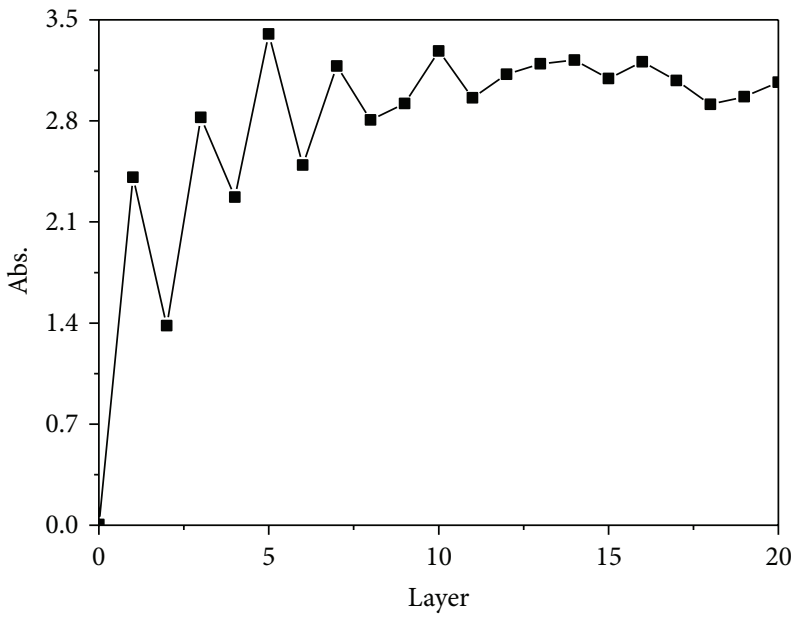

(b)

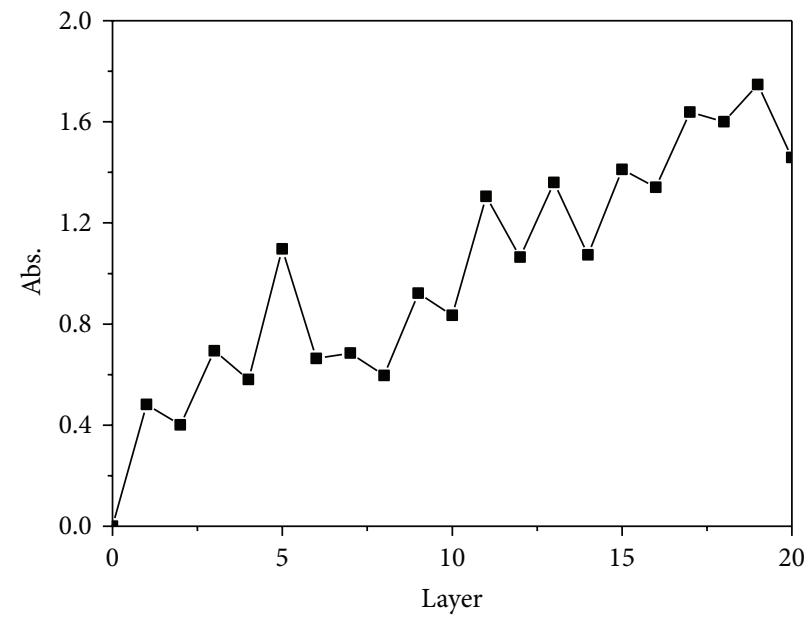

(c)

FIGURE 3: (a) Schematic illustration to show self-assembly process of poly(CM- $\beta$-CD)/PLL multilayer with drug by incorporating drug into PLL solution. (b) UV absorbance of hydrogel at $285 \mathrm{~nm}$ for orfloxacin as a function of layers. (c) UV absorbance of hydrogel at $255 \mathrm{~nm}$ for puerarin as a function of layers.

cumulative drug release amount was obtained according to drug concentration and volume.

2.7. Statistical Analysis. Data were analyzed using the $t$-test for differences. Results are reported as means \pm standard deviation. The significant level was set at $P<0.05$.

\section{Result and Discussion}

Poly $(\mathrm{CM}-\beta-\mathrm{CD})$ was obtained by epichlorohydrin crosslinking of $-\mathrm{OH}$ group as shown in Figure 1(a), which was characterized by IR spectrum in Figure 1(b). It is found that the peak of $1743 \mathrm{~cm}^{-1}$ belonging to ester and the peak of $1028 \mathrm{~cm}^{-1}$ belonging to ether emerged in the IR spectrum of poly $(C M-\beta-C D)$, which indicated that CM- $\beta-C D$ had been successfully cross-linked. At the same time, the peak of $1630 \mathrm{~cm}^{-1}$ belonging to $-\mathrm{COOH}$ group still existed in the spectrum of poly(CM- $\beta-\mathrm{CD})$, which indicated that poly $(\mathrm{CM}-\beta-\mathrm{CD})$ was a polyanion.

Figure 2(a) showed multilayer self-assembled process on hydrogel with the alternate deposition of poly(CM$\beta$-CD) and PLL by manual dip-coating. The electrostatic 


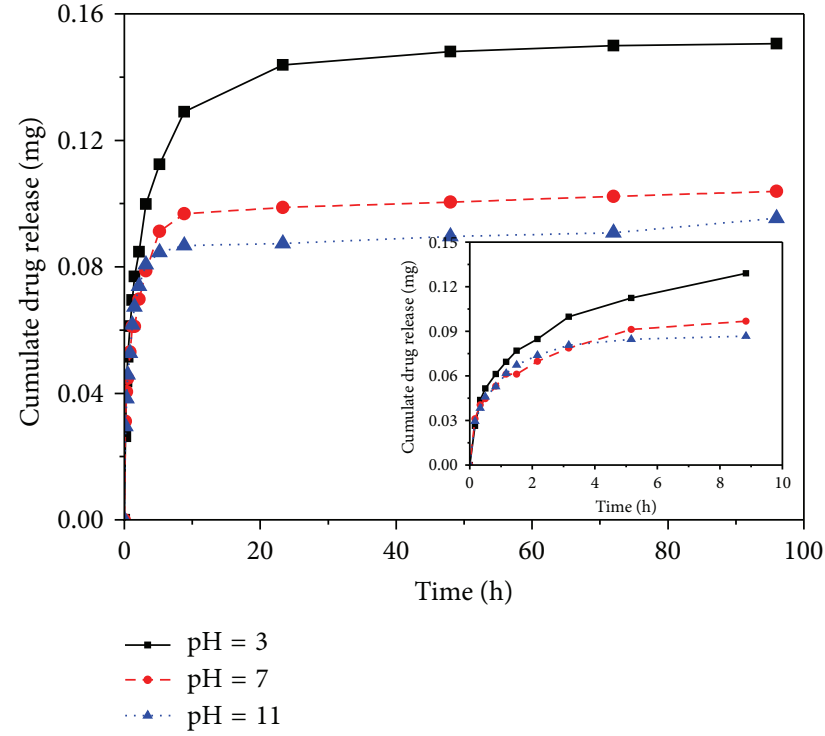

(a)

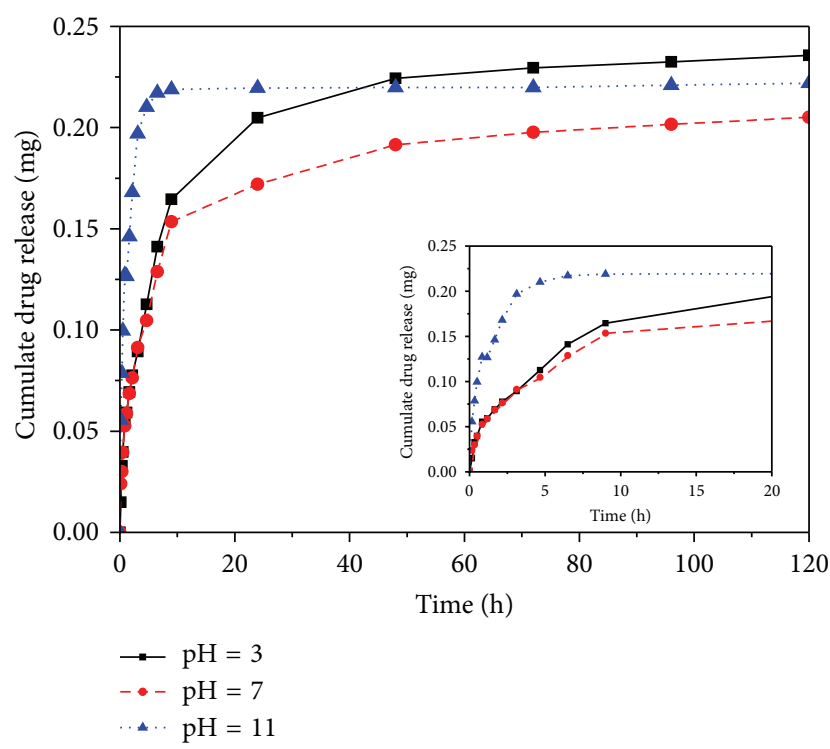

(b)

Figure 4: (a) The cumulate orfloxacin release from drug loaded poly(CM- $\beta$-CD)/PLL(drug $)_{20}$ multilayer in different medium at $37^{\circ} \mathrm{C}$. (b) The cumulate puerarin release from drug loaded poly $(\mathrm{CM}-\beta-\mathrm{CD}) / \mathrm{PLL}(\mathrm{drug})_{20}$ multilayer in different medium at $37^{\circ} \mathrm{C}$.

interaction between these groups is the main driving force to form poly(CM- $\beta$-CD)/PLL multilayer on the hydrogel. Since hydrogel surface was modified by HA, it was negatively charged surface. Thus, positively charged PLL was anchored on the negatively charged hydrogel to form the first layer during the self-assembled process. The hydrophilicity of hydrogel was further investigated by contact angle measurements, as shown in Figure 2(b). The contact angle increased after two PLL layers and two poly $(\mathrm{CM}-\beta-\mathrm{CD})$ layers were assembled on the hydrogel and decreased little after another PLL layer was assembled. Subsequent assembling of PLL and poly $(\mathrm{CM}-\beta-\mathrm{CD})$ layers exhibited similar trends showing low and high contact angles until the layer number reached 14. Then the contact angle was not varied with assembled layers. However, all the variations showed no significant difference. Light transmittance of hydrogel was detected by UV spectrum in Figure 2(c). Although the transparence of copolymer hydrogel decreased after 20 poly $(\mathrm{CM}-\beta-\mathrm{CD}) / \mathrm{PLL}$ layers were assembled on the hydrogel, larger than $83 \%$ light could transmit the hydrogel when wavelength was larger than $350 \mathrm{~nm}$, which means hydrogel had acceptable transparence in the visible region.

Drug was loaded into multilayer by two methods during the assembly procedure. Drugs were first incorporated in PLL assembly solution. As it is known to us, orfloxacin was an anion charged drug, which was bonded to polycation PLL by electrostatic attraction as well as intermolecular forces, while puerarin was a nonionic drug, which was bonded to polycation PLL by intermolecular forces, such as hydrogen bond between - $\mathrm{OH}$ group of puerarin and $-\mathrm{NH}_{2}$ groups of PLL. In order to track the drug loading assembly procedure, the absorbance at $285 \mathrm{~nm}$ for orfloxacin or $255 \mathrm{~nm}$ for puerarin of hydrogel with different layers was recorded in Figures 3(b) and 3(c). When the first PLL layer was deposited on the hydrogel, the absorbance of hydrogel at $285 \mathrm{~nm}$ increased to 2.5 because of orfloxacin incorporation (Figure 3(b)). When the second poly $(\mathrm{CM}-\beta$ $\mathrm{CD}$ ) layer was deposited on the hydrogel, the absorbance of hydrogel at $285 \mathrm{~nm}$ decreased to 1.4 because of the mask of outer layer as well as diffusion of orfloxacin into the poly(CM$\beta$-CD) assembly solution. Subsequent assembling of PLL and poly $(\mathrm{CM}-\beta-\mathrm{CD})$ layers exhibited similar trends showing high and low absorbance at $285 \mathrm{~nm}$ until the layer number reached 10. Then the absorbance of hydrogel at $285 \mathrm{~nm}$ stabilized to 2.8-3.0, perhaps because of saturated orfloxacin concentration in the multilayer of hydrogel. When the drug was puerarin, the variation of absorbance with assembled layer for puerarin showed similar trend to that for orfloxacin at initial 5-6 poly $(\mathrm{CM}-\beta-\mathrm{CD}) /$ PLL layers (Figure 3(c)). Differently, this trend continued for puerarin until 20 layers were all assembled on the hydrogel. At the same time, the absorbance of hydrogel at $255 \mathrm{~nm}$ presented overall upward trend with the increase of assembled layer and reached 1.4-1.6. These results indicated that the saturated puerarin concentration in the multilayer of hydrogel was unreached.

The drug release behaviors of hydrogel with the above drug loaded multilayer in different medium were shown in Figure 4. At the initial $1 \mathrm{~h}$, about $0.05 \mathrm{mg}$ orfloxacin was released fast from drug loaded hydrogel (Figure 4(a)). Then orfloxacin release behavior at acid environment $(\mathrm{pH}=$ 3) was different from that at neutral environment $(\mathrm{pH}=$ 7) and alkaline environment $(\mathrm{pH}=11)$. The orfloxacin was slowly released from hydrogel in the following $10 \mathrm{~h}$ at acid environment; the balanced cumulate orfloxacin release amount reached $0.15 \mathrm{mg}$. While the orfloxacin was slowly released from hydrogel in the following $10 \mathrm{~h}$ at neutral environment or alkaline environment, the balanced cumulate orfloxacin release amount reached $0.10 \mathrm{mg}$. Orfloxacin 

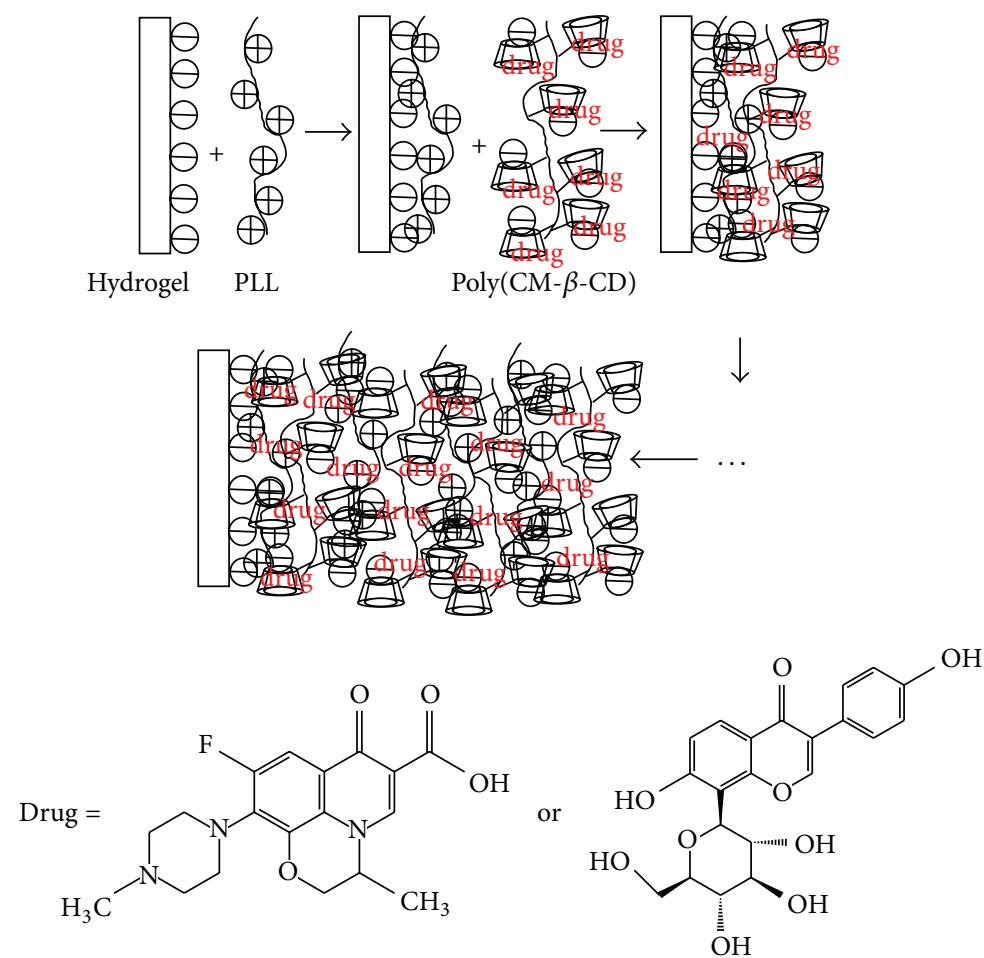

(a)

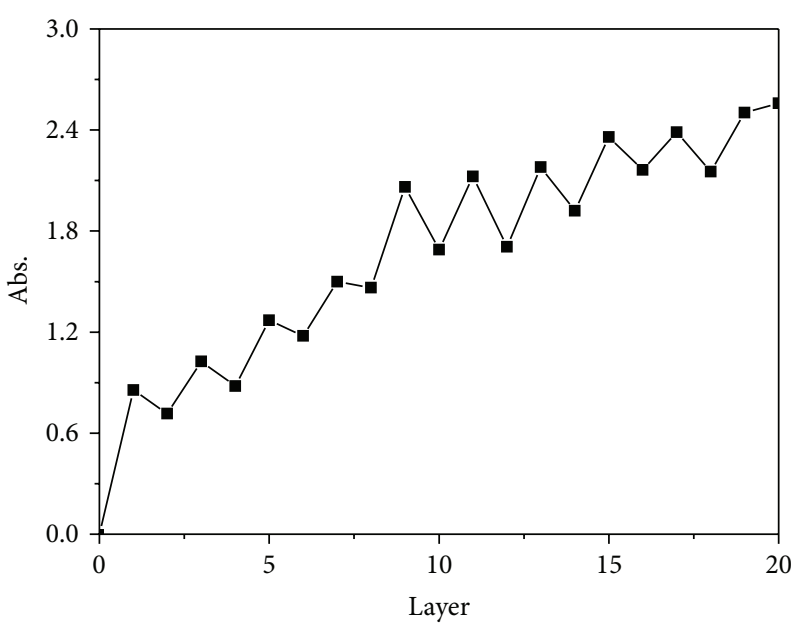

(b)

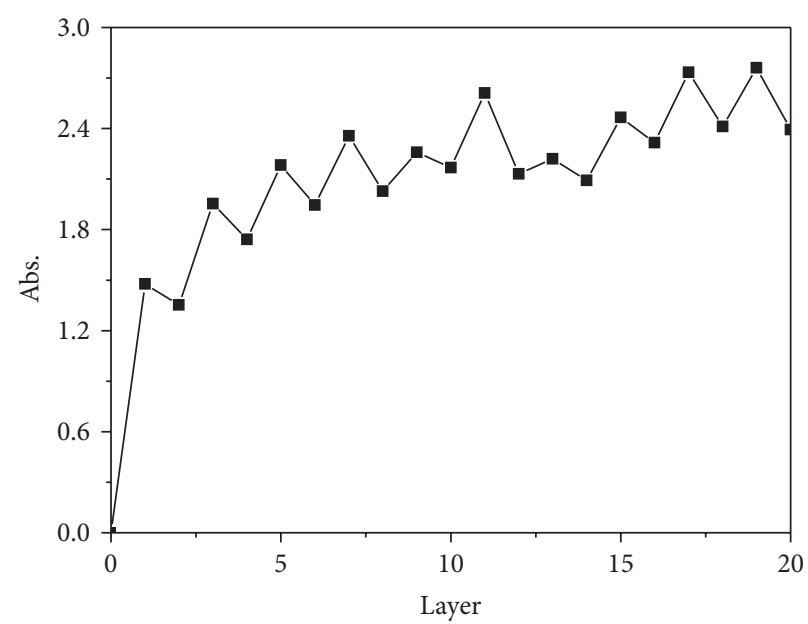

(c)

Figure 5: (a) Schematic illustration to show self-assembly process of poly(CM- $\beta$-CD)/PLL multilayer with drug by incorporating drug into poly(CM- $\beta$-CD) solution. (b) UV absorbance of hydrogel at $285 \mathrm{~nm}$ for orfloxacin as a function of layers. (c) UV absorbance of hydrogel at $255 \mathrm{~nm}$ for puerarin as a function of layers.

was nearly in neutral state at acid environment. Thus the interaction between orfloxacin and multilayer reduced at acid environment, which led to larger cumulate orfloxacin release amount. As for puerarin, the release behavior was different from that of orfloxacin (Figure 4(b)). Puerarin was gradually released from hydrogel in $20 \mathrm{~h}$ at neutral environment and alkaline environment after the initial burst release. The final cumulate puerarin release amount at neutral environment and alkaline environment reached $0.20 \mathrm{mg}$ and $0.24 \mathrm{mg}$, respectively. However, puerarin was quickly released from hydrogel in the initial $1 \mathrm{~h}$ at acid environment. As discussed above, puerarin might be bonded to PLL by hydrogen bond, which would weaken in acid environment. Thus puerarin exhibited a burst release at acid environment.

Drugs were secondly incorporated in poly $(\mathrm{CM}-\beta-\mathrm{CD})$ assembly solution. Drugs were assembled into multilayer by the $\beta$-CD/drug inclusion complexes, which was also a noncovalent interaction of $\beta$-CD and drug molecules. The absorbance at $285 \mathrm{~nm}$ for orfloxacin or $255 \mathrm{~nm}$ for puerarin of hydrogel with different layers was recorded in 


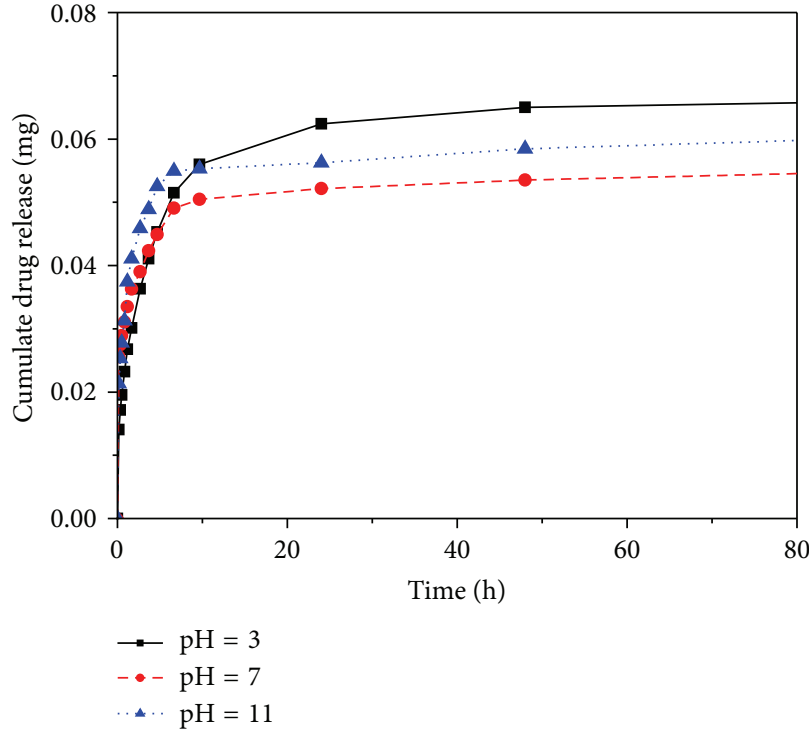

(a)

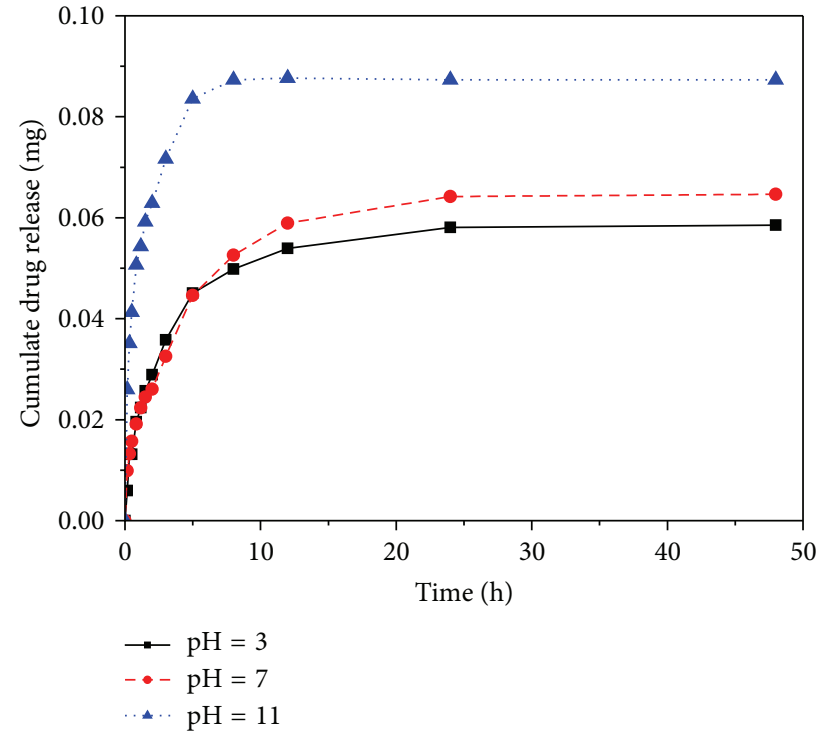

(b)

Figure 6: (a) The cumulate orfloxacin release from drug loaded poly $(\mathrm{CM}-\beta-\mathrm{CD})(\mathrm{drug}) / \mathrm{PLL}_{20}$ multilayer in different medium at $37^{\circ} \mathrm{C}$. (b) The cumulate puerarin release from drug loaded poly $(\mathrm{CM}-\beta-\mathrm{CD})(\mathrm{drug}) / \mathrm{PLL}_{20}$ multilayer in different medium at $37^{\circ} \mathrm{C}$.

Figures 5(b) and 5(c). The assembling of poly $(\mathrm{CM}-\beta-\mathrm{CD})$ and PLL layers exhibited similar trends showing relatively high and low absorbance at $285 \mathrm{~nm}$ (Figure 5(b)). The absorbance at $285 \mathrm{~nm}$ presented overall upward trend with the increase of assembled layer and reached about 2.4. The variation of absorbance with assembled layer for puerarin showed similar trend to that for orfloxacin and the balanced absorbance reached about 2.7 (Figure 5(c)). It was also found from Figures 3(c) and 5(c) that the global absorbance of puerarin loaded multilayer by incorporating puerarin in poly $(\mathrm{CM}-\beta-\mathrm{CD})$ solution was much larger than that by incorporating puerarin in PLL solution, which indicated that loaded puerarin amount in the multilayer from poly $(\mathrm{CM}-\beta$ $\mathrm{CD})$ solution was much larger than that from PLL solution. These results inferred that the interaction coming from $\beta$-CD/puerarin inclusion complexes was larger than the interaction of hydrogen bonds between PLL and puerarin.

The drug release behaviors of hydrogel with the above drug loaded multilayer in different medium were shown in Figure 6. Orfloxacin was gradually released from drug loaded hydrogel in $10 \mathrm{~h}$ at acid environment, neutral environment, or alkaline environment after first burst release (Figure 6(a)). But the balanced orfloxacin release amount only reached $0.06 \mathrm{mg}$. As for puerarin, the release behavior was similar to that of orfloxacin at acid environment or neutral environment and the balanced puerarin release amount only reached $0.06 \mathrm{mg}$ (Figure 6(b)). The balanced puerarin release amount at acid environment reached $0.09 \mathrm{mg}$. Though the loaded puerarin amount in the multilayer through the second method was larger than that through the first method, the cumulate puerarin amount through the second method was much smaller than that through the first method, which indicated that many puerarin molecules stably existed in the multilayer due to relatively stable structure of $\beta-\mathrm{CD} /$ puerarin inclusion complexes. Therefore, we can conclude from these results that the drug loading and releasing behaviors can be adjusted by loading method, the structure of drug, and released environment.

\section{Conclusion}

A polyanion of poly $(\mathrm{CM}-\beta-\mathrm{CD})$ was successfully synthesized by epichlorohydrin cross-linking of CM- $\beta$-CD. The poly $(\mathrm{CM}-\beta-\mathrm{CD})$ and PLL were alternately deposited on the hydrogel by LBL self-assembly technique. With the deposition of poly $(\mathrm{CM}-\beta-\mathrm{CD}) / \mathrm{PLL}$ multilayer, the contact angle of hydrogel varied regularly and slightly. Larger than $83 \%$ light could transmit the hydrogel with poly $(\mathrm{CM}-\beta-$ $\mathrm{CD}) / \mathrm{PLL}_{20}$ multilayer. Orfloxacin and puerarin could be gradually loaded into multilayer during the self-assembly procedure by incorporating drugs into PLL solution, which was tracked by the largest drug absorbance of UV spectrum. The loaded orfloxacin in the multilayer reached balance after 10 layers of polyelectrolyte were assembled on the hydrogel, while the loaded puerarin in the multilayer did not reach balance until 20 layers of polyelectrolyte were assembled on the hydrogel. The loaded drug could gradually be released from multilayer in $20 \mathrm{~h}$ either for orfloxacin or for puerarin. The $\mathrm{pH}$ of released medium influenced the drug release behavior. Orfloxacin and puerarin could also be gradually loaded into multilayer during the self-assembly procedure by incorporating drugs into poly $(\mathrm{CM}-\beta-\mathrm{CD})$ solution, and the loaded puerarin amount was larger than that by incorporating drugs into PLL solution. The loaded drug could gradually be released from multilayer in $10 \mathrm{~h}$ either. But the balanced released drug amount is much smaller than that by 
incorporating drugs into PLL solution. Moreover, the $\mathrm{pH}$ of released medium also influenced the drug release behavior.

\section{Conflict of Interests}

The authors declare that there is no conflict of interests regarding the publication of this paper.

\section{Acknowledgments}

This study is financially supported by the Natural Science Foundation of China (51103066), the Qing Lan Project, and the Research Fund of Jinling Institute of Technology in China (Jit-n-201203, Jit-n-201101).

\section{References}

[1] W. Liu, M. Griffith, and F. Li, "Alginate microsphere-collagen composite hydrogel for ocular drug delivery and implantation," Journal of Materials Science: Materials in Medicine, vol. 19, no. 11, pp. 3365-3371, 2008.

[2] X. Chen, X. Li, Y. Zhou et al., "Chitosan-based thermosensitive hydrogel as a promising ocular drug delivery system: preparation, characterization, and in vivo evaluation," Journal of Biomaterials Applications, vol. 27, no. 4, pp. 391-402, 2012.

[3] C. Lu, R. B. Yoganathan, M. Kociolek, and C. Allen, "Hydrogel containing silica shell cross-linked micelles for ocular drug delivery," Journal of Pharmaceutical Sciences, vol. 102, no. 2, pp. 627-637, 2013.

[4] J. Yu, X. Xu, F. Yao et al., "In situ covalently cross-linked PEG hydrogel for ocular drug delivery applications," International Journal of Pharmaceutics, vol. 470, no. 1-2, pp. 151-157, 2014.

[5] C. Lu, X. Wang, G. Wu et al., "An injectable and biodegradable hydrogel based on poly $(\alpha, \beta$-aspartic acid $)$ derivatives for localized drug delivery," Journal of Biomedical Materials Research Part A, vol. 102, no. 3, pp. 628-638, 2014.

[6] G. R. Bardajee and Z. Hooshyar, "One-pot synthesis of biocompatible superparamagnetic iron oxide nanoparticles/hydrogel based on salep: characterization and drug delivery," Carbohydrate Polymers, vol. 101, no. 1, pp. 741-751, 2014.

[7] G. Jiang, J. Sun, and F. Ding, "PEG-g-chitosan thermosensitive hydrogel for implant drug delivery: cytotoxicity, in vivo degradation and drug release," Journal of Biomaterials Science, Polymer Edition, vol. 25, no. 3, pp. 241-256, 2014.

[8] C.-C. Peng and A. Chauhan, "Extended cyclosporine delivery by silicone-hydrogel contact lenses," Journal of Controlled Release, vol. 154, no. 3, pp. 267-274, 2011.

[9] H. J. Jung, M. Abou-Jaoude, B. E. Carbia, C. Plummer, and A. Chauhan, "Glaucoma therapy by extended release of timolol from nanoparticle loaded silicone-hydrogel contact lenses," Journal of Controlled Release, vol. 165, no. 1, pp. 82-89, 2013.

[10] L. C. Bengani, J. Leclerc, and A. Chauhan, "Lysozyme transport in p-HEMA hydrogel contact lenses," Journal of Colloid and Interface Science, vol. 386, no. 1, pp. 441-450, 2012.

[11] M. Robitaille, J. Shi, S. McBride, and K. T. Wan, "Mechanical performance of hydrogel contact lenses with a range of power under parallel plate compression and central load," Journal of the Mechanical Behavior of Biomedical, vol. 2, pp. 259-264, 2013.

[12] M. Bettuelli, S. Trabattoni, M. Fagnola, S. Tavazzi, L. Introzzi, and S. Farris, "Surface properties and wear performances of siloxane-hydrogel contact lenses," Journal of Biomedical Materials Research. Part B Applied Biomaterials, vol. 101, no. 8, pp. 1585-1593, 2013.

[13] X. H. Hu and D. Li, "Facile way to synthesise hydrogel contact lenses with good performance for ophthalmic drug delivery," Materials Technology, vol. 28, no. 4, pp. 192-198, 2013.

[14] X. H. Hu, X. X. Chen, H. P. Tan, D. Li, Y. S. Zhang, and C. B. Pan, "Preparation of biocompatible graphene oxide composite hydrogel to deliver ophthalmic drugs," Journal of Macromolecular Science, Part A: Pure and Applied Chemistry, vol. 50, no. 12, pp. 1201-1208, 2013.

[15] X. H. Hu, J. Qiu, H. P. Tan, D. Li, and X. H. Ma, "Synthesis and characterization of cyclodextrin-containing hydrogel for ophthalmic drugs delivery," Journal of Macromolecular Science, Part A, vol. 50, pp. 983-990, 2013.

[16] A. E. El Haitami, J.-S. Thomann, L. Jierry et al., "Covalent layerby-layer assemblies of polyelectrolytes and homobifunctional spacers," Langmuir, vol. 26, no. 14, pp. 12351-12357, 2010.

[17] R. Gill, M. Mazhar, O. Felix, and G. Decher, "Covalent layerby-layer assembly and solvent memory of multilayer films from homobifunctional poly(dimethylsiloxane)," Angewandte Chemie International Edition, vol. 49, no. 35, pp. 6116-6119, 2010.

[18] Z. Zhao, L. Yin, G. Yuan, and L. Wang, "Layer-by-layer assembly of two temperature-responsive homopolymers at neutral $\mathrm{pH}$ and the temperature-dependent solubility of the multilayer film," Langmuir, vol. 28, no. 5, pp. 2704-2709, 2012.

[19] Y. Guo, W. Geng, and J. Sun, "Layer-by-layer deposition of polyelectrolyte-polyelectrolyte complexes for multilayer film fabrication," Langmuir, vol. 25, no. 2, pp. 1004-1010, 2009.

[20] Q. Tang, J. Wu, X. Sun, Q. Li, and J. Lin, "Layer-by-layer self-assembly of conducting multilayer film from poly(sodium styrenesulfonate) and polyaniline," Journal of Colloid and Interface Science, vol. 337, no. 1, pp. 155-161, 2009.

[21] D.-G. Yu, W.-C. Lin, and M.-C. Yang, "Surface modification of poly(L-lactic acid) membrane via layer-by-layer assembly of silver nanoparticle-embedded polyelectrolyte multilayer," Bioconjugate Chemistry, vol. 18, no. 5, pp. 1521-1529, 2007.

[22] X. H. Hu, H. P. Tan, D. Li, and M. Y. Gu, "Surface functionalisation of contact lenses by CS/HA multilayer film to improve its properties and deliver drugs," Materials Technology, vol. 29, no. 1, pp. 8-13, 2014.

[23] T. R. Thatiparti and H. A. von Recum, "Cyclodextrin complexation for affinity-based antibiotic delivery," Macromolecular Bioscience, vol. 10, no. 1, pp. 82-90, 2010.

[24] T. R. Thatiparti, A. J. Shoffstall, and H. A. von Recum, "Cyclodextrin-based device coatings for affinity-based release of antibiotics," Biomaterials, vol. 31, no. 8, pp. 2335-2347, 2010.

[25] J. Chen, W.-L. Lu, W. Gu et al., "Drug-in-cyclodextrin-inliposomes: a promising delivery system for hydrophobic drugs," Expert Opinion on Drug Delivery, vol. 11, no. 4, pp. 565-577, 2014.

[26] R. J. Glisoni, M. J. García-Fernández, M. Pino et al., “ $\beta$ Cyclodextrin hydrogels for the ocular release of antibacterial thiosemicarbazones," Carbohydrate Polymers, vol. 93, no. 2, pp. 449-457, 2013.

[27] J. Xu, X. Li, and F. Sun, "Cyclodextrin-containing hydrogels for contact lenses as a platform for drug incorporation and release," Acta Biomaterialia, vol. 6, no. 2, pp. 486-493, 2010.

[28] J.-F. Rosa dos Santos, C. Alvarez-Lorenzo, M. Silva et al., "Soft contact lenses functionalized with pendant cyclodextrins for controlled drug delivery," Biomaterials, vol. 30, no. 7, pp. 13481355, 2009. 
[29] X. Gong, J. Yang, L. Han, and C. Gao, "Influence of drying time of polyelectrolyte multilayers on the compression-induced pattern formation," Langmuir, vol. 24, no. 24, pp. 13925-13933, 2008.

[30] X. Gong, L. Han, Y. Yue, J. Gao, and C. Gao, "Influence of assembly $\mathrm{pH}$ on compression and $\mathrm{Ag}$ nanoparticle synthesis of polyelectrolyte multilayers," Journal of Colloid and Interface Science, vol. 355, no. 2, pp. 368-373, 2011.

[31] X. Gong, L. Han, J. Gao, and C. Gao, "Stability of polyelectrolyte multilayer micropatterns in response to post-treatments," Colloids and Surfaces A: Physicochemical and Engineering Aspects, vol. 396, pp. 299-304, 2012.

[32] X. Gong and C. Gao, "Influence of salt on assembly and compression of PDADMAC/PSSMA polyelectrolyte multilayers," Physical Chemistry Chemical Physics, vol. 11, no. 48, pp. 1157711586, 2009. 

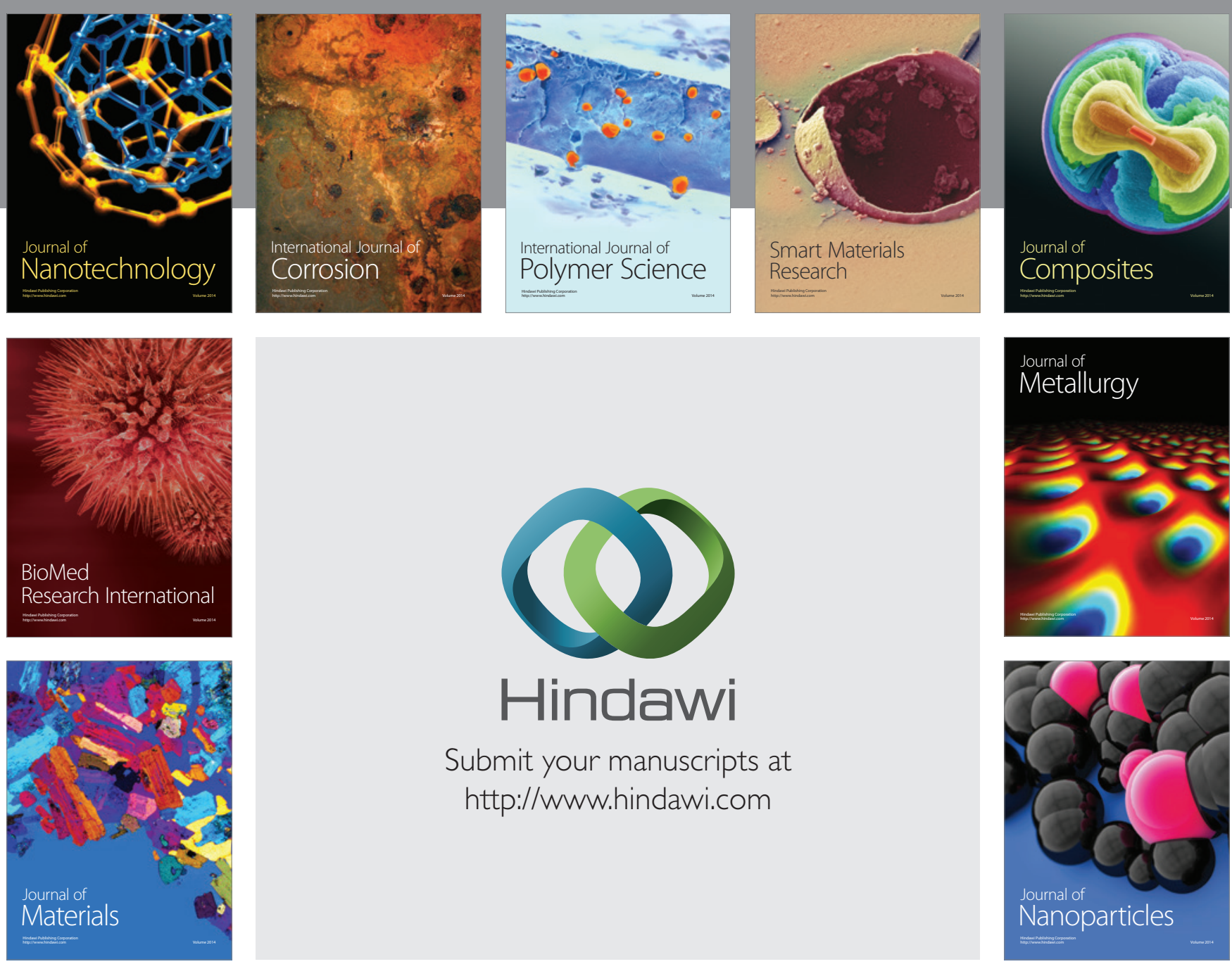

Submit your manuscripts at http://www.hindawi.com
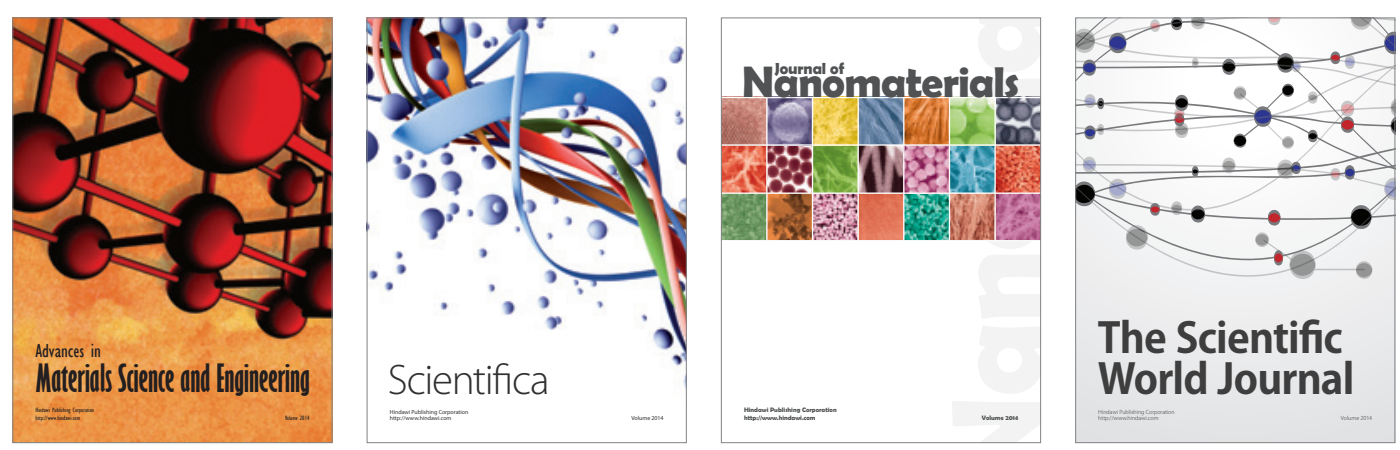

\section{The Scientific World Journal}
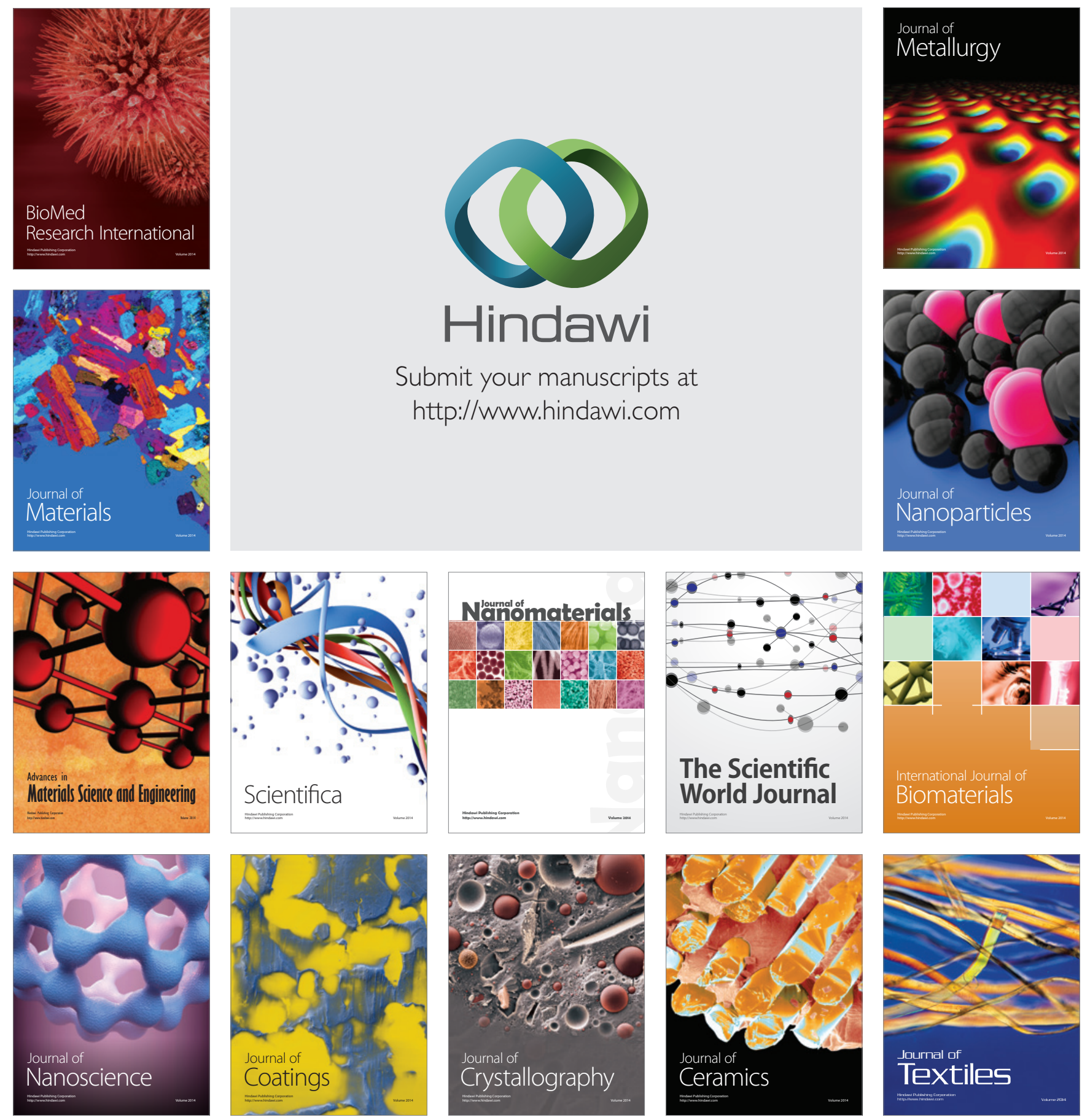